\title{
Fast radar detection method based on two-dimensional trilinear autocorrelation function for maneuvering target with jerk motion
}

\author{
Zhongying Liang, ${ }^{a}$ Yanyan Li $\odot,{ }^{b} *$ Jinping Niu, ${ }^{b}$ Lin Wang $\odot$, \\ XiaoXuan Chen, ${ }^{b}$ and Ling Wang ${ }^{a}$

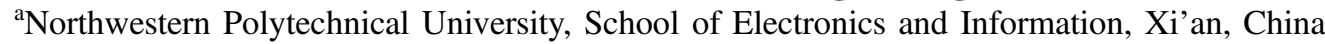 \\ ${ }^{b}$ Northwest University, School of Information Science and Technology, Xi'an, China
}

\begin{abstract}
We focus on the range migration (RM) and Doppler frequency migration (DFM) corrections in the long-time coherent integration, and a fast detection method based on twodimensional trilinear autocorrelation function is proposed for the maneuvering target with jerk motion. This proposed method can integrate the echoes' energy into peaks in a three-dimensional parameter space coherently and estimate the target's radial range, acceleration, and jerk simultaneously by the peak detection technique. Then through the estimations of radial range, acceleration, and jerk, the radial velocity can be obtained through one-dimensional parameter searching. Finally, RM and DFM can be compensated simultaneously, and the target can be detected through the constant false alarm technique. This proposed method can strike a good balance between the computational complexity and detection performance. Experiments with the simulation and real measured radar data are conducted to verify the proposed method. (C) The Authors. Published by SPIE under a Creative Commons Attribution 4.0 Unported License. Distribution or reproduction of this work in whole or in part requires full attribution of the original publication, including its DOI. [DOI: 10.1117/1.JRS.15.026508]
\end{abstract}

Keywords: maneuvering target; parameter estimation; range migration; Doppler frequency migration; two-dimensional trilinear autocorrelation function.

Paper 210040L received Jan. 22, 2021; accepted for publication Apr. 15, 2021; published online May 3, 2021.

\section{Introduction}

Maneuvering target detection and motion parameters estimation are important applications of radar, therefore, the study on these aspects has received wide attention in the past decades. ${ }^{1-10}$ With the development of science and technique, the target's radar cross section becomes lower and lower, and the radar detection performance is affected. In order to improve the radar detection performance without any change of radar systems, prolonging the integration time is usually required. ${ }^{11}$ Unfortunately, for maneuvering target, during the long integration time, the range migration (RM) and Doppler frequency migration (DFM) usually appear, which will make the conventional coherent integration algorithm, i.e., moving target detection (MTD) ${ }^{12}$ suffer from performance loss, even failure. Therefore, to obtain better detection performance, it is necessary to compensate the RM and DFM effectively.

The keystone transform (KT), ${ }^{13,14}$ Radon-Fourier transform (RFT) ${ }^{15,16}$ scaled inverse Fourier transform, ${ }^{17}$ frequency-domain deramp $\mathrm{KT},{ }^{18}$ and frequency autocorrelation function-Lv's distribution ${ }^{8}$-based methods are typical in compensating the RM and DFM induced by the target's radial velocity and acceleration. However, for the highly maneuvering target, i.e., with jerk motion, these above-mentioned methods will suffer from detection performance. To overcome this problem, the generalized RFT (GRFT) ${ }^{19}$ which completes the coherent integration via jointly searching along range cell, radial velocity, acceleration, and jerk directions, is proposed. The GRFT can achieve a good detection performance, but the brute-force parameters searching will increase the computational complexity greatly.

*Address all correspondence to Yanyan Li, liyanyan_xd@163.com 
Liang et al.: Fast radar detection method based on two-dimensional trilinear autocorrelation function...

To reduce the computational complexity, some detection methods based on nonlinear autocorrelation functions (AFs) are proposed. These methods can be divided into two categories: the AFs constructed in the range frequency domain and slow time domain, respectively. In Ref. 20, the method that defines the AF with respect to the range frequency domain, can balance the computational complexity and detection performance well. The KT generalized dechirp process (KT-GDP), ${ }^{9}$ KT cubic phase function (KT-CPF), ${ }^{9}$ product scaled integrated cubic phase function (PSICPF) ${ }^{10}$ adjacent cross-correlation function-Lv's distribution (ACCF-LVD) ${ }^{21}$ and the ACCF iteratively ${ }^{21}$-based methods construct $\mathrm{AFs}$ in the slow time domain, these methods can reduce computational complexity greatly compared with the GRFT. For the KT-GDP and KT-CPF, the incoherent integration in fold factor searching makes these two methods to be sensitive to the noise. In the PSICPF-based method, because of the modulus square operation on the result obtained after scaled non-uniform fast Fourier transform operation, the phase information is discarded, and the resolution of parameter estimation will be affected. For the ACCF-LVD and ACCF iteratively based methods, although the integration processes are coherent, these two methods have poor detection performance under low signal-to-noise ratio (SNR) environment due to the ACCF operations.

Based on the consideration of improving the balance between the computational complexity and detection performance for the methods constructing the AFs in the slow time domain, a coherent detection method based on two-dimensional (2D) trilinear autocorrelation function (TAF) is proposed in this paper.

The layout of this paper is as follows. In Sec. 2, the signal model of the maneuvering target with jerk motion is given. In Sec. 3, the principle of this proposed method is introduced in detail. Cross-term suppression performance, detection performance, and computational complexity are analyzed and compared with other representative methods in Sec. 4. An experiment with the raw radar data is carried out to validate the effectiveness of the proposed method in Sec. 5. Finally, the conclusion of this paper is given.

\section{Signal Model for Maneuvering Target with Jerk Motion}

Assume the linear frequency modulated signal is transmitted by the radar, which can be stated as

$$
s_{t}(t)=\operatorname{rect}\left(t / T_{p}\right) \exp \left(j \pi \gamma t^{2}\right),
$$

where rect( $\cdot)$ is a rectangle windows function, $t$ and $T_{P}$ denote the fast time and pulse duration, respectively. $\gamma=B / T_{P}$ represents the frequency modulation rate, and $B$ is the signal bandwidth. After the demodulation, the target's $2 \mathrm{D}$ baseband echoes can be expressed as ${ }^{19}$

$$
\begin{aligned}
X_{r}\left(t, t_{n}\right) & =S_{r}\left(t, t_{n}\right)+Z_{r}\left(t, t_{n}\right) \\
& =\sum_{i=1}^{I} A_{i} \operatorname{rect}\left[\frac{t-2 R_{i}\left(t_{n}\right) / c}{T_{p}}\right] \exp \left\{j \pi \gamma\left[t-\frac{2 R_{i}\left(t_{n}\right)}{c}\right]^{2}\right\} \exp \left[-j \frac{4 \pi R_{i}\left(t_{n}\right)}{\lambda}\right]+Z_{r}\left(t, t_{n}\right),
\end{aligned}
$$

where $t_{n}$ is the slow time, $A_{i}$ is the complex amplitude of the $i$ th target, and $c$ and $\lambda$ are the light speed and wavelength, respectively. $Z_{r}\left(t, t_{n}\right)$ stands for the additive complex white noise. $R_{i}\left(t_{n}\right)=r_{i}+v_{i} t_{n}+a_{i} t_{n}^{2} / 2+k_{i} t_{n}^{3} / 6$ is the time-varying range between the $i$ 'th moving target and radar, $r_{i}, v_{i}, a_{i}$, and $k_{i}$ denote the initial radial range, radial velocity, acceleration, and jerk of the $i$ 'th target, separately. $I$ is the number of targets.

After performing the pulse compression (PC) operation, we have

$$
\begin{aligned}
X_{c i}\left(t, t_{n}\right)= & \sum_{i=1}^{I} A_{c i} \operatorname{sinc}\left\{B\left[t-\frac{2\left(r_{i}+v_{i} t_{n}+a_{i} t_{n}^{2} / 2+k_{i} t_{n}^{3} / 6\right)}{c}\right]\right\} \\
& \times \exp \left(-j 4 \pi \frac{r_{i}+v_{i} t_{n}+a_{i} t_{n}^{2} / 2+k_{i} t_{n}^{3} / 6}{\lambda}\right)+Z_{c i}\left(t, t_{n}\right),
\end{aligned}
$$


where $A_{c i}$ is the $i$ 'th target's complex amplitude after PC operation. $\operatorname{sinc}(x)$ is a sinc function. $Z_{c i}\left(t, t_{n}\right)$ is the noise after PC operation.

\section{Principle of the Proposed 2D TAF-Based Method}

It can be seen from Eq. (3), the range profile alignment is disturbed by the RM and DFM, thus the energy integration is difficult to be realized. In order to solve this problem, a method, which can compensate the RM and DFM simultaneously, is proposed.

After performing the fast Fourier transform (FFT) operation on Eq. (3) along the $t$ axis, we can obtain the signal form in the range frequency and slow time domain as follows:

$$
\begin{aligned}
X\left(F_{r}, t_{n}\right)= & S\left(F_{r}, t_{n}\right)+Z\left(F_{r}, t_{n}\right) \\
= & \sum_{i=1}^{I} A_{0 i} \operatorname{rect}\left(\frac{F_{r}}{B}\right) \exp \left(-j 4 \pi r_{i} \frac{F_{c}+F_{r}}{c}\right) \\
& \times \exp \left[-j \frac{4 \pi}{\lambda} \frac{F_{c}+F_{r}}{F_{c}}\left(v_{i} t_{n}+a_{i} t_{n}^{2} / 2+k_{i} t_{n}^{3} / 6\right)\right]+Z\left(F_{r}, t_{n}\right),
\end{aligned}
$$

where $A_{0 i}$ is the $i$ 'th target's complex amplitude obtained after the FFT operation, $F_{r}$ denotes the range frequency with respect to the fast time, $F_{c}$ is the carrier frequency, and $Z\left(F_{r}, t_{n}\right)$ is the noise. Obviously, $S\left(F_{r}, t_{n}\right)$ is a $2 \mathrm{D}$ quadratic frequency modulated signal. In order to compensate the RM and DFM, the targets' motion parameters need to be estimated.

We first define a bilinear $\mathrm{AF}$ as follows:

$$
R_{0}\left(F_{r}, t_{n}, \tau_{n}\right)=X\left(F_{r}, t_{n}+\tau_{n}+\tau_{0}\right) X\left(F_{r}, t_{n}-\tau_{n}-\tau_{0}\right)
$$

where $\tau_{n}$ and $\tau_{0}$ denote the lag-time variable and constant delay, respectively. Substituting Eq. (4) into Eq. (5), we can get

$$
\begin{aligned}
R_{0}\left(F_{r}, t_{n}, \tau_{n}\right)= & \sum_{i=1}^{I} A_{0 i}^{2} \operatorname{rect}\left(\frac{F_{r}}{B}\right) \exp \left(-j 8 \pi r_{i} \frac{F_{c}+F_{r}}{c}\right) \\
& \underbrace{\times \exp \left\{-j \frac{4 \pi}{\lambda} \frac{F_{c}+F_{r}}{F_{c}}\left[\left(2 v_{i} t_{n}+a_{i} t_{n}^{2}+k_{i} t_{n}^{3} / 3\right)+\left(a_{i}+k_{i} t_{n}\right)\left(\tau_{n}+\tau_{0}\right)^{2}\right]\right\}}_{\text {autoterms }} \\
& +\underbrace{R_{0, c \text {-terms }}\left(F_{r}, t_{n}, \tau_{n}\right)}_{\text {cross terms }}+\underbrace{R_{0, \text { n-noise }}\left(F_{r}, t_{n}, \tau_{n}\right)}_{\text {noise terms }} .
\end{aligned}
$$

Note that Eq. (6) can be rearranged as

$$
\begin{aligned}
R_{0}\left(F_{r}, t_{n}, \tau_{n}\right)= & \sum_{i=1}^{I} A_{0 i}^{2} \operatorname{rect}\left(\frac{F_{r}}{B}\right) \exp \left(-j 8 \pi r_{i} \frac{F_{c}+F_{r}}{c}\right) \\
& \times \exp \left\{-j \frac{4 \pi}{\lambda} \frac{F_{c}+F_{r}}{F_{c}}\left(a_{i}+k_{i} t_{n}\right)\left[\left(\tau_{n}+\tau_{0}\right)^{2}-t_{n}^{2}\right]\right\} \\
& \times \exp \left\{-j \frac{4 \pi}{\lambda} \frac{F_{c}+F_{r}}{F_{c}}\left[v_{i}\left(2 t_{n}\right)+a_{i}\left(2 t_{n}\right)^{2} / 2+k_{i}\left(2 t_{n}\right)^{3} / 6\right]\right\} \\
& +R_{0, c \text {-terms }}\left(F_{r}, t_{n}, \tau_{n}\right)+R_{0, n \text {-noise }}\left(F_{r}, t_{n}, \tau_{n}\right) .
\end{aligned}
$$

To compensate the third exponential term of Eq. (7), we define a 2D TAF as follows:

$$
R\left(F_{r}, t_{n}, \tau_{n}\right)=X\left(F_{r}, t_{n}+\tau_{n}+\tau_{0}\right) X\left(F_{r}, t_{n}-\tau_{n}-\tau_{0}\right) X^{*}\left(F_{r}, 2 t_{n}\right),
$$

where * represents the complex conjugate.

Substituting Eq. (4) into Eq. (8), we can obtain 


$$
\begin{aligned}
R\left(F_{r}, t_{n}, \tau_{n}\right)= & \sum_{i=1}^{I} A_{0 i}^{3} \operatorname{rect}\left(\frac{F_{r}}{B}\right) \exp \left(-j 4 \pi r_{i} \frac{F_{c}+F_{r}}{c}\right) \\
& \underbrace{\times \exp \left\{-j 2 \pi\left(\frac{F_{c}+F_{r}}{F_{c}}\right) \frac{2\left(a_{i}+k_{i} t_{n}\right)}{\lambda}\left[\left(\tau_{n}+\tau_{0}\right)^{2}-t_{n}^{2}\right]\right\}}_{\text {autoterms }} \\
& +\underbrace{R_{c \text {-terms }}\left(F_{r}, t_{n}, \tau_{n}\right)}_{\text {cross terms }}+\underbrace{R_{n \text {-noise }}\left(F_{r}, t_{n}, \tau_{n}\right)}_{\text {noise terms }} .
\end{aligned}
$$

Then apply the NUFFT operation on Eq. (9) along the $\tau_{n}$ axis, we have

$$
\begin{aligned}
R_{1}\left(F_{r}, t_{n}, F_{\tau_{n}}\right)= & \int_{\tau_{n}} R\left(F_{r}, t_{n}, \tau_{n}\right) \exp \left[-j 2 \pi\left(\frac{F_{c}+F_{r}}{F_{c}}\right) F_{\tau_{n}}\left(\tau_{n}+\tau_{0}\right)^{2}\right] \mathrm{d} \tau_{n} \\
= & \sum_{i=1}^{I} A_{1 i} \operatorname{rect}\left(\frac{F_{r}}{B}\right) \exp \left(-j 4 \pi r_{i} \frac{F_{c}+F_{r}}{c}\right) \\
& \times \exp \left\{j 2 \pi\left(\frac{F_{c}+F_{r}}{F_{c}}\right) \frac{2\left(a_{i}+k_{i} t_{n}\right)}{\lambda} t_{n}^{2}\right\} \delta\left[F_{\tau_{n}}+\frac{2\left(a_{i}+k_{i} t_{n}\right)}{\lambda}\right] \\
& +R_{1, c \text {-terms }}\left(F_{r}, t_{n}, F_{\tau_{n}}\right)+R_{1, n \text {-terms }}\left(F_{r}, t_{n}, F_{\tau_{n}}\right),
\end{aligned}
$$

where $A_{1 i}$ is the $i$ 'th target's complex amplitude obtained after the NUFFT operation along the $\tau_{n}$ axis, $F_{\tau_{n}}$ is the frequency domain corresponding to $\tau_{n}$. With the sampling property of Dirac delta function, Eq. (10) can be approximated as

$$
\begin{aligned}
R_{1}\left(F_{r}, t_{n}, F_{\tau_{n}}\right)= & \sum_{i=1}^{I} A_{1 i} \operatorname{rect}\left(\frac{F_{r}}{B}\right) \exp \left(-j 4 \pi r_{i} \frac{F_{c}+F_{r}}{c}\right) \\
& \times \exp \left\{-j 2 \pi\left(\frac{F_{c}+F_{r}}{F_{c}}\right) F_{\tau_{n}} t_{n}^{2}\right\} \delta\left[F_{\tau_{n}}+\frac{2\left(a_{i}+k_{i} t_{n}\right)}{\lambda}\right] \\
& +R_{1, c \text {-terms }}\left(F_{r}, t_{n}, F_{\tau_{n}}\right)+R_{1, n \text {-terms }}\left(F_{r}, t_{n}, F_{\tau_{n}}\right) .
\end{aligned}
$$

In Eq. (11), the second exponential item can be compensated as follows:

$$
\begin{aligned}
R_{2}\left(F_{r}, t_{n}, F_{\tau_{n}}\right)= & R_{1}\left(F_{r}, t_{n}, F_{\tau_{n}}\right) \exp \left\{j 2 \pi\left(\frac{F_{c}+F_{r}}{F_{c}}\right) F_{\tau_{n}} t_{n}^{2}\right\} \\
= & \sum_{i=1}^{I} A_{1 i} \operatorname{rect}\left(\frac{F_{r}}{B}\right) \exp \left(-j 4 \pi r_{i} \frac{F_{c}+F_{r}}{c}\right) \delta\left[F_{\tau_{n}}+\frac{2\left(a_{i}+k_{i} t_{n}\right)}{\lambda}\right] \\
& +R_{2, c \text {-terms }}\left(F_{r}, t_{n}, F_{\tau_{n}}\right)+R_{2, n \text {-terms }}\left(F_{r}, t_{n}, F_{\tau_{n}}\right) .
\end{aligned}
$$

Thereafter, performing the inverse FFT (IFFT) operation on Eq. (12) along the $F_{r}$ axis, we can get

$$
\begin{aligned}
R_{3}\left(t, t_{n}, F_{\tau_{n}}\right)= & \operatorname{IFFT}_{F_{r}}\left[R_{2}\left(F_{r}, t_{n}, F_{\tau_{n}}\right)\right] \\
= & \sum_{i=1}^{I} A_{2 i} \operatorname{sinc}\left[B\left(t-\frac{2 r_{i}}{c}\right)\right] \exp \left(-j 2 \pi \frac{2 r_{i}}{\lambda}\right) \delta\left[F_{\tau_{n}}+\frac{2\left(a_{i}+k_{i} t_{n}\right)}{\lambda}\right] \\
& +R_{3, c \text {-terms }}\left(t, t_{n}, F_{\tau_{n}}\right)+R_{3, n \text {-terms }}\left(t, t_{n}, F_{\tau_{n}}\right)
\end{aligned}
$$

where $A_{2 i}$ is the $i$ 'th target's complex amplitude obtained after the IFFT operation along the $F_{r}$ axis, and $t$ is the time domain corresponding to $F_{r}$. It can be seen from Eq. (13), the energy accumulation along the inclined line $F_{\tau_{n}}=-\left(a_{i}+k_{i} t_{n}\right) / \lambda$, which can be accomplished through the Hough transform. ${ }^{1,22}$ In order to reduce the energy loss in Hough transform, the interpolation technique with a factor 4 is adopted for $F_{\tau_{n}}$. According to the principle of Hough transform, the coherent integration along the inclined line can be expressed as 


$$
\begin{aligned}
R_{4}\left(t, \hat{a}_{i}, \hat{k}_{i}\right)= & \sum_{t_{n}} R_{3}\left\{\left[t, t_{n},-\frac{2}{\lambda}\left(\hat{a}_{i}+\operatorname{round}\left(\hat{k}_{i} t_{n} / \Delta F_{\tau_{n}}\right) \Delta F_{\tau_{n}}\right)\right]\right\} \\
= & \sum_{i=1}^{I} A_{3 i} \operatorname{sinc}\left[B\left(t-\frac{2 r_{i}}{c}\right)\right] \exp \left(-j 2 \pi \frac{2 r_{i}}{\lambda}\right) \delta\left(\hat{a}_{i}-a_{i}\right) \delta\left(\hat{k}_{i}-k_{i}\right) \\
& +R_{4, c \text {-terms }}\left(t, \hat{a}_{i}, \hat{k}_{i}\right)+R_{4, n \text {-terms }}\left(t, \hat{a}_{i}, \hat{k}_{i}\right),
\end{aligned}
$$

where $A_{3 i}$ is the $i$ 'th target's complex amplitude obtained after the coherent integration along $F_{\tau_{n}}=-\left(a_{i}+k_{i} t_{n}\right) / \lambda$, round $(\cdot)$ denotes the operation that rounds to nearest integer, and $\Delta F_{\tau_{n}}$ is the frequency interval of $F_{\tau_{n}}$. round $\left(\hat{k}_{i} t_{n} / \Delta F_{\tau_{n}}\right) \Delta F_{\tau_{n}}$ can be precalculated and stored, therefore, Eq. (14) can be accomplished though the addition operation. Obviously, only when the $i$ 'th target's acceleration and jerk are matched with $\hat{a}_{i}$ and $\hat{k}_{i}$, the energy can be accumulated completely. Each target generates a sole peak, according to the peak coordinate, $r_{i}, a_{i}$, and $k_{i}$ can be estimated. Utilizing these estimations, $v_{i}$ is estimated via searching in the target's velocity space, which only requires one-dimensional (1D) maximization.

With the estimated motion parameters, we construct a compensation function as follows:

$$
Y\left(F_{r}, t_{n} ; \hat{v}_{i}, \hat{a}_{i}, \hat{k}_{i}\right)=\exp \left[j 4 \pi \frac{F_{r}}{c} \hat{v}_{i} t_{n}+j 4 \pi \frac{F_{c}+F_{r}}{c}\left(\hat{a}_{i} t_{n}^{2} / 2+\hat{k}_{i} t_{n}^{3} / 6\right)\right] .
$$

Multiplying Eq. (15) with Eq. (4), the RM and DFM of the $i$ 'th target will be compensated simultaneously. Thereafter, the range frequency IFFT and azimuth FFT operations are performed to realize the coherent integration. Finally, the constant false alarm detection technique is employed to accomplish the target detection.

Based on analyses above, the detailed flowchart of the proposed method is shown in Fig. 1.

\section{Performance of the Proposed Method}

\subsection{Cross-Term Suppression Performance}

According to the definition of 2D TAF, we know the cross terms will appear. Here in order to analyze the cross-term suppression performance of the proposed method, we consider two

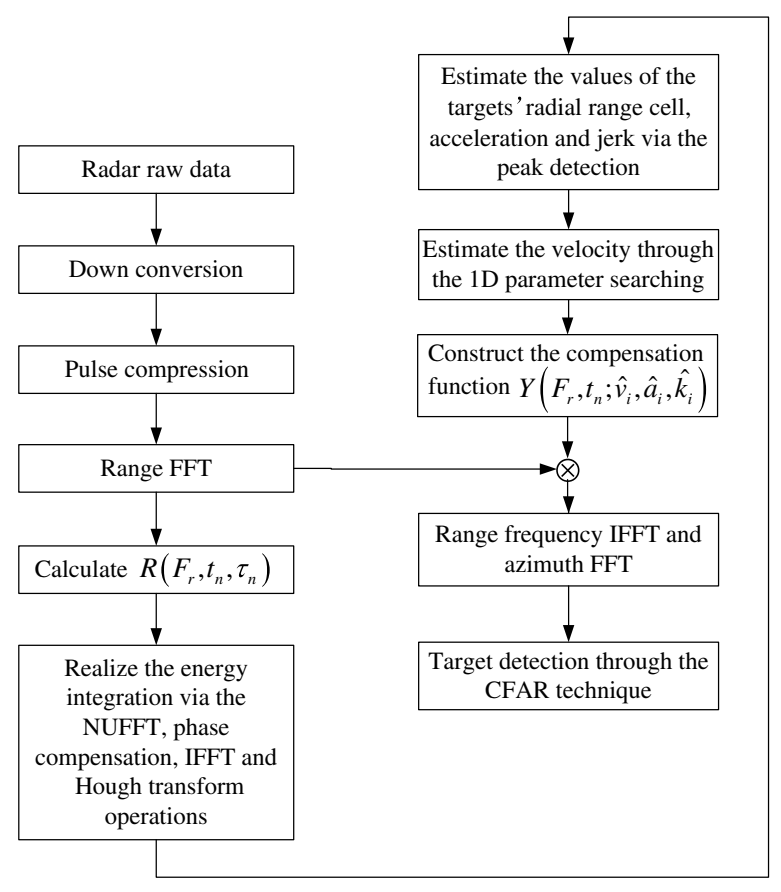

Fig. 1 Flowchart of the proposed method. 
noise-free targets' echoes, i.e., $S_{1}\left(F_{r}, t_{n}\right)$ and $S_{2}\left(F_{r}, t_{n}\right) . A_{01}$ and $A_{02}$ denote the echoes' amplitudes, $r_{1}, r_{2}, v_{1}, v_{2}, a_{1}, a_{2}, k_{1}$, and $k_{2}$ denote the initial radial ranges, radial velocities, accelerations, and jerks of these two targets, separately:

$$
\begin{aligned}
R_{c \text {-terms }}\left(F_{r}, t_{n}, \tau_{n}\right)= & S_{1}\left(F_{r}, t_{n}+\tau_{n}+\tau_{0}\right) S_{1}\left(F_{r}, t_{n}-\tau_{n}-\tau_{0}\right) S_{2}^{*}\left(F_{r}, 2 t_{n}\right) \\
& +S_{1}\left(F_{r}, t_{n}+\tau_{n}+\tau_{0}\right) S_{2}\left(F_{r}, t_{n}-\tau_{n}-\tau_{0}\right) S_{1}^{*}\left(F_{r}, 2 t_{n}\right) \\
& +S_{1}\left(F_{r}, t_{n}+\tau_{n}+\tau_{0}\right) S_{2}\left(F_{r}, t_{n}-\tau_{n}-\tau_{0}\right) S_{2}^{*}\left(F_{r}, 2 t_{n}\right) \\
& +S_{2}\left(F_{r}, t_{n}+\tau_{n}+\tau_{0}\right) S_{1}\left(F_{r}, t_{n}-\tau_{n}-\tau_{0}\right) S_{1}^{*}\left(F_{r}, 2 t_{n}\right) \\
& +S_{2}\left(F_{r}, t_{n}+\tau_{n}+\tau_{0}\right) S_{1}\left(F_{r}, t_{n}-\tau_{n}-\tau_{0}\right) S_{2}^{*}\left(F_{r}, 2 t_{n}\right) \\
& +S_{2}\left(F_{r}, t_{n}+\tau_{n}+\tau_{0}\right) S_{2}\left(F_{r}, t_{n}-\tau_{n}-\tau_{0}\right) S_{1}^{*}\left(F_{r}, 2 t_{n}\right) .
\end{aligned}
$$

The first term of Eq. (16) has the form of

$$
\begin{aligned}
R_{c \text {-terms }, 1}\left(F_{r}, t_{n}, \tau_{n}\right)= & S_{1}\left(F_{r}, t_{n}+\tau_{n}+\tau_{0}\right) S_{1}\left(F_{r}, t_{n}-\tau_{n}-\tau_{0}\right) S_{2}^{*}\left(F_{r}, 2 t_{n}\right) \\
= & A_{01} \operatorname{rect}\left(\frac{F_{r}}{B}\right) \exp \left(-j 4 \pi r_{1} \frac{F_{c}+F_{r}}{c}\right) \\
& \times \exp \left\{-j \frac{4 \pi}{\lambda} \frac{F_{c}+F_{r}}{F_{c}}\left(a_{1}+k_{1} t_{n}\right)\left[\left(\tau_{n}+\tau_{0}\right)^{2}-t_{n}^{2}\right]\right\} S_{1}\left(F_{r}, 2 t_{n}\right) S_{2}^{*}\left(F_{r}, 2 t_{n}\right) .
\end{aligned}
$$

Performing the NUFFT operation along the $\tau_{n}$ axis follows:

$$
\begin{aligned}
Q_{1}\left(F_{r}, t_{n}, F_{\tau_{n}}\right)= & \int_{\tau_{n}} R_{c \text {-terms }, 1}\left(F_{r}, t_{n}, \tau_{n}\right) \exp \left[-j 2 \pi\left(\frac{F_{c}+F_{r}}{F_{c}}\right) F_{\tau_{n}}\left(\tau_{n}+\tau_{0}\right)^{2}\right] \mathrm{d} \tau_{n} \\
= & A_{01}^{\prime} \operatorname{rect}\left(\frac{F_{r}}{B}\right) \exp \left(-j 4 \pi r_{1} \frac{F_{c}+F_{r}}{c}\right) \\
& \times \exp \left\{j 2 \pi\left(\frac{F_{c}+F_{r}}{F_{c}}\right) \frac{2\left(a_{1}+k_{1} t_{n}\right)}{\lambda} t_{n}^{2}\right\} \delta\left[F_{\tau_{n}}+\frac{2\left(a_{1}+k_{1} t_{n}\right)}{\lambda}\right] \\
& \times S_{1}\left(F_{r}, 2 t_{n}\right) S_{2}^{*}\left(F_{r}, 2 t_{n}\right),
\end{aligned}
$$

where $A_{01}^{\prime}$ denotes the amplitude after the NUFFT operation. It can be seen only when $r_{1}=r_{2}$, $v_{1}=v_{2}, a_{1}=a_{2}$, and $k_{1}=k_{2}$, Eq. (18) can be accumulated into a peak by the later phase compensation and IFFT operation.

The second term of Eq. (16) can be expressed as

$$
\begin{aligned}
R_{c \text {-terms }, 2}\left(F_{r}, t_{n}, \tau_{n}\right)= & S_{1}\left(F_{r}, t_{n}+\tau_{n}+\tau_{0}\right) S_{2}\left(F_{r}, t_{n}-\tau_{n}-\tau_{0}\right) S_{1}^{*}\left(F_{r}, 2 t_{n}\right) \\
= & A_{01}^{2} A_{02} \operatorname{rect}\left(\frac{F_{r}}{B}\right) \exp \left(-j 4 \pi r_{2} \frac{F_{c}+F_{r}}{c}\right) \\
& \times \exp \left[-j \frac{4 \pi}{\lambda} \frac{F_{c}+F_{r}}{F_{c}}\left(v_{1}-v_{2}\right)\left(t_{n}+\tau_{n}+\tau_{0}\right)\right] \\
& \times \exp \left[-j \frac{4 \pi}{\lambda} \frac{F_{c}+F_{r}}{F_{c}}\left(a_{1}-a_{2}\right)\left(t_{n} / 2+\tau_{n}+\tau_{0}\right) t_{n}\right] \\
& \times \exp \left[-j \frac{2 \pi}{\lambda} \frac{F_{c}+F_{r}}{F_{c}}\left(k_{1}-k_{2}\right)\left[t_{n}^{3}+3\left(\tau_{n}+\tau_{0}\right) t_{n}^{2}+\left(\tau_{n}+\tau_{0}\right)^{2}\right] / 3\right] \\
& \times \exp \left[-j \frac{2 \pi}{\lambda} \frac{F_{c}+F_{r}}{F_{c}}\left[\left(a_{1}+a_{2}\right)+\left(k_{1}+k_{2}\right) t_{n}\right]\left(\tau_{n}+\tau_{0}\right)^{2}\right] .
\end{aligned}
$$

Obviously, only when $v_{1}=v_{2}, a_{1}=a_{2}$, and $k_{1}=k_{2}$, Eq. (19) can be accumulated into a straight line by the NUFFT operation, and then a peak locates at the same point as the autoterm in the 3D parameter space. 
Liang et al.: Fast radar detection method based on two-dimensional trilinear autocorrelation function...

Table 1 Radar parameters and targets' motion parameters

\begin{tabular}{|c|c|c|c|c|c|}
\hline \multicolumn{2}{|c|}{ Carrier frequency } & $1 \mathrm{GHz}$ & \multicolumn{2}{|c|}{ Sampling frequency } & $5 \mathrm{MHz}$ \\
\hline \multicolumn{2}{|c|}{ Bandwidth } & $5 \mathrm{MHz}$ & \multicolumn{2}{|c|}{ Effective echo pulses $N$} & 512 \\
\hline \multicolumn{2}{|c|}{ PRF } & $256 \mathrm{~Hz}$ & \multicolumn{2}{|c|}{ Effective integration time } & $2 \mathrm{~s}$ \\
\hline \multicolumn{2}{|c|}{ Pulse width } & $80 \mu \mathrm{s}$ & \multicolumn{2}{|l|}{ Constant delay } & $1 \mathrm{~s}$ \\
\hline & Range cell unit & Amplitude & Velocity $(\mathrm{m} / \mathrm{s})$ & Acceleration $\left(\mathrm{m} / \mathrm{s}^{2}\right)$ & Jerk $\left(m / s^{3}\right)$ \\
\hline T1 & 100th & 1 & 850 & 9 & 6 \\
\hline T2 & 160th & 1 & -600 & -6 & -3 \\
\hline T3 & 160th & 1 & 900 & 9 & 3 \\
\hline
\end{tabular}

In Eq. (16), the sixth term has the same form as the first term, the third, fourth, and fifth terms have the same form as the second term. Thus we can conclude that the cross terms do not influence the autoterms detection.

To illustrate the effectiveness and cross-term suppression performance of the proposed method, an example is given below.

Example 1: Three targets T1, T2, and T3 are considered, in order to describe simply, here, the noise is assumed to be absent. The radar parameters and targets' motion parameters are listed in Table 1. The simulation results are shown in Fig. 2.

Via performing the 2D TAF, NUFFT operation on Eq. (9) along the $\tau_{n}$ axis, and phase compensation on Eq. (11), Fig. 2(a) shows the slow time-scaled Doppler frequency distribution at $F_{r}=0 \mathrm{~Hz}$, i.e., $R_{2}\left(0, t_{n}, F_{\tau_{n}}\right)$. In this plane, it can be seen that three straight lines corresponding to three autoterms are accumulated. Then after the IFFT operation along $F_{r}$ axis and energy accumulation along the inclined line $F_{\tau_{n}}=-\left(a_{i}+k_{i} t_{n}\right) / \lambda$, we obtain $R_{4}\left(t, \hat{a}_{i}, \hat{k}_{i}\right)$. Figs. 2(b) and 2(c) show the acceleration-jerk distributions of $R_{4}\left(t, \hat{a}_{i}, \hat{k}_{i}\right)$ for $t=20 \mu \mathrm{s}$ and $t=32 \mu \mathrm{s}$, respectively. Obviously, the autoterms have been integrated, whereas the cross terms are dispersed and even can be ignored. The range cell-jerk distributions of $R_{4}\left(t, \hat{a}_{i}, \hat{k}_{i}\right)$ for $\hat{a}_{i}=-6 \mathrm{~m} / \mathrm{s}^{2}$ and $\hat{a}_{i}=9 \mathrm{~m} / \mathrm{s}^{2}$ as given in Figs. 2(d) and 2(e) also demonstrate the above-mentioned conclusion. Through the peak detection technique, the radial ranges, accelerations, and jerks of these three targets can be estimated precisely, then we can estimate the radial velocities via 1D parameter searching, and the result is shown in Fig. 2(f).

\subsection{Detection Performance}

In this section, an experiment is utilized to evaluate the detection performances of the proposed method, GRFT, method in Ref. 20, ACCF-LVD, and ACCF iteratively based detection methods. A single target located at the 100'th range cell is considered, and the radial velocity, acceleration, and jerk are set as $900 \mathrm{~m} / \mathrm{s}, 9 \mathrm{~m} / \mathrm{s}^{2}$, and $3 \mathrm{~m} / \mathrm{s}^{3}$, respectively. The radar parameters are set as: $F_{c}=1 \mathrm{GHz}, B=10 \mathrm{MHz}, \mathrm{PRF}=200 \mathrm{~Hz}$, the sampling frequency is $10 \mathrm{MHz}$, and the number of effective integration pulses is 200. Complex white Gaussian noise is added before PC operation. The input SNRs are $[-50: 1: 30] \mathrm{dB}$, and 200 trials are performed for each input SNR value. The detection probabilities with false alarm probability $P_{\mathrm{fa}}=10^{-4}$ are shown in Fig. 3. Because of many input SNRs, the points that represent the detection probabilities will be dense, to see more clearly, the results are plotted at 2-dB interval along the SNR-IN axis in Fig. 3.

The GRFT has the best detection performance due to the four-dimensional parameters searching. For the method in Ref. 20, the ACCF iteratively, ACCF-LVD, and the proposed method, some output SNR losses are caused by the nonlinear AFs, therefore, their detection performances are worse than the GRFT. Higher order AF will result in more detection 
Liang et al.: Fast radar detection method based on two-dimensional trilinear autocorrelation function...

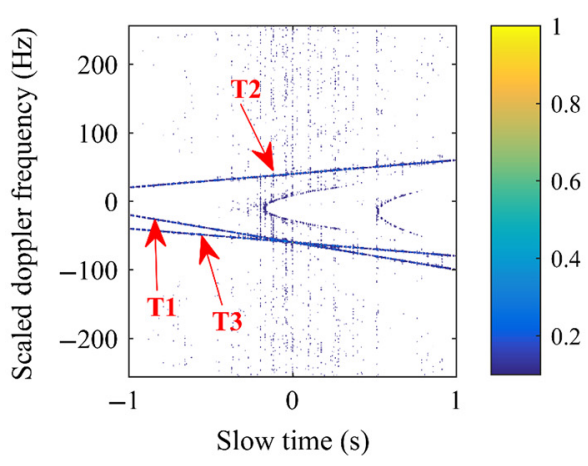

(a)

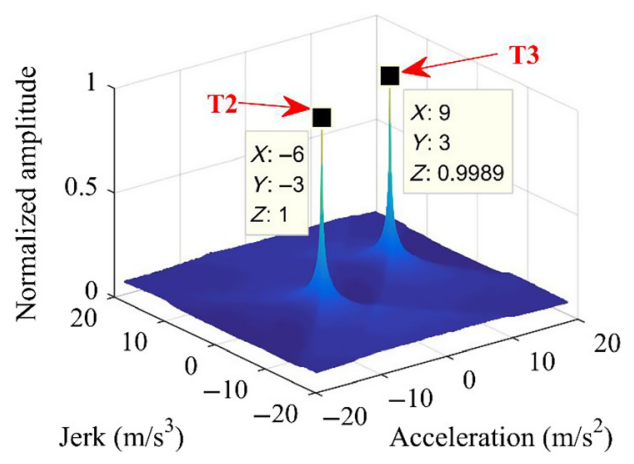

(c)

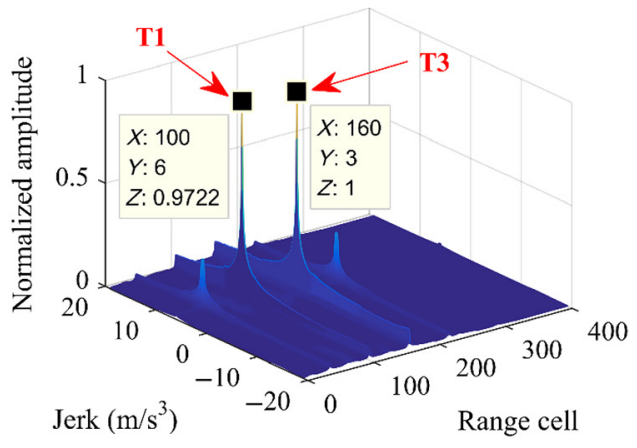

(e)

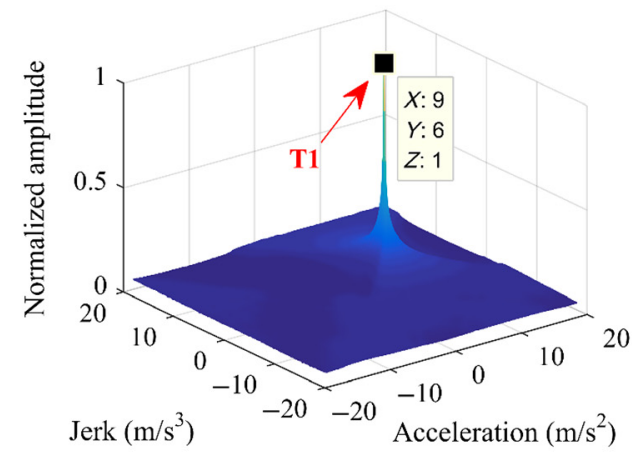

(b)

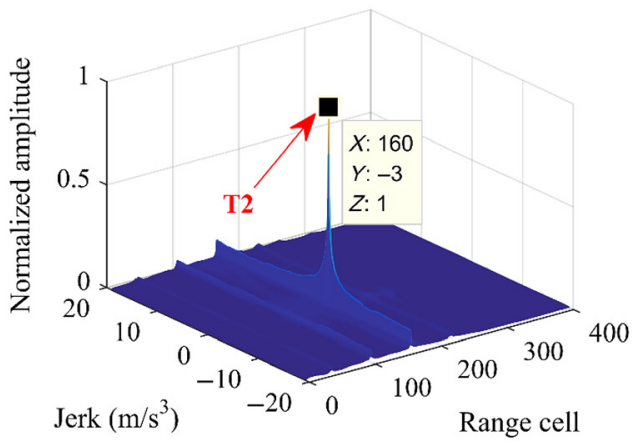

(d)

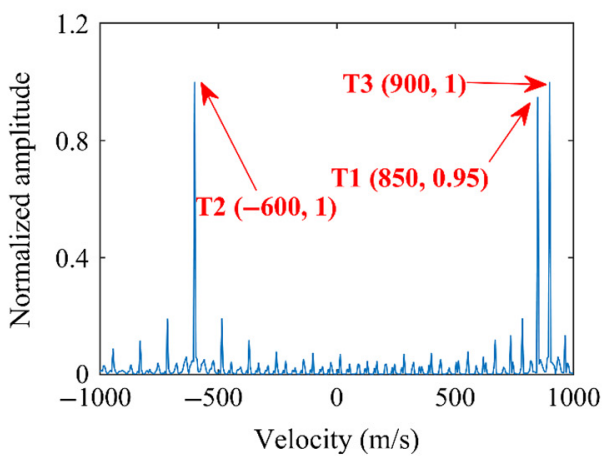

(f)

Fig. 2 Simulation results of example 1: (a) slow time-scaled Doppler frequency distribution; (b) cross section of $R_{4}\left(t, \hat{a}_{i}, \hat{k}_{i}\right)$ for $t=20 \mu \mathrm{s}$; (c) cross section of $R_{4}\left(t, \hat{a}_{i}, \hat{k}_{i}\right)$ for $t=32 \mu \mathrm{s}$; (d) cross section of $R_{4}\left(t, \hat{a}_{i}, \hat{k}_{i}\right)$ for $\hat{a}_{i}=-6 \mathrm{~m} / \mathrm{s}^{2}$; (e) cross section of $R_{4}\left(t, \hat{a}_{i}, \hat{k}_{i}\right)$ for $\hat{a}_{i}=9 \mathrm{~m} / \mathrm{s}^{2}$; and (f) the estimated velocities for these three targets.

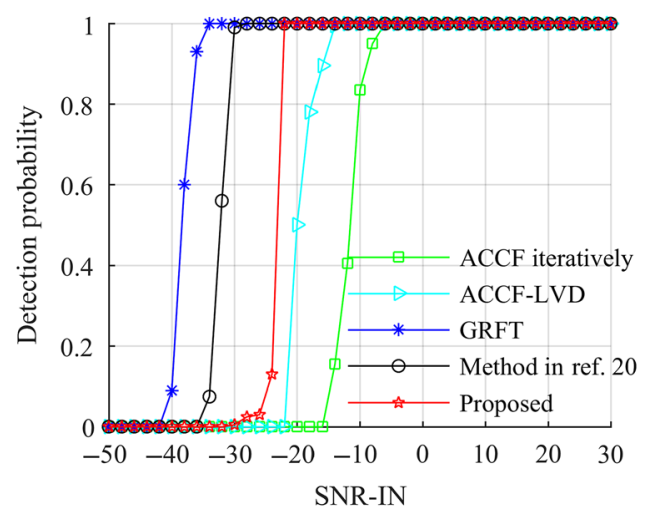

Fig. 3 Comparison results of the detection probability. 
performance loss. The AFs of the method in Ref. 20, ACCF-LVD, ACCF iteratively, and the proposed method are second order, forth order, forth order, and third order, respectively. Due to the lower order AF, the method in Ref. 20 has better detection than the other three methods.

For the methods defining the AFs with respect to the slow time domain, because the AF's order of the proposed 2D TAF is lower than those of the ACCF-LVD and ACCF iteratively based method, the detection performance of the proposed method is much better than the ACCF iteratively and ACCF-LVD-based detection methods as shown in Fig. 3.

\subsection{Computational Complexity Analysis}

This section is devoted to compare the computational complexity between the proposed method and other representative methods. Denote the numbers of range cells and integration pulses by $M$ and $N$, the searching numbers of radial velocity, acceleration, and jerk are $N_{v}, N_{a}$, and $N_{k}$, respectively.

According to Refs. 19-21, the computational complexities of the GRFT, method in Ref. 20, ACCF-LVD, and ACCF iteratively based methods are $\left[O\left(N_{v} N_{a} N_{k} M N\right)\right]$, $\left[O\left(M^{2} N+5 M N \log _{2} N+2 N M \log _{2} M+N^{2} \log _{2} N\right)\right], \quad\left[O\left(6 M N^{2} \log _{2} N+N M \log _{2} M\right)\right]$, and $\left[O\left(3 M N \log _{2} N+3 N M \log _{2} M\right)\right]$, respectively.

For the proposed method, its main procedures have the following steps. (1) The calculation of $R\left(F_{r}, t_{n}, \tau_{n}\right)$ is $\left[O\left(2 M N^{2}\right)\right]$. (2) Because the interpolation technique with a factor 4 for $F_{\tau_{n}}$ is adopted in the implementation of this method, the length of NUFFT should be $4 N$, and the calculation of the NUFFT operation along the $\tau_{n}$ axis is $\left[O\left(8 M N^{2} \log _{2} N+16 M N^{2}\right)\right]$. (3) The calculation of compensating the second exponential item of Eq. (12) is $\left[O\left(4 M N^{2}\right)\right]$. (4) The IFFT operation along the $F_{r}$ axis $\left[O\left(N^{2} M \log _{2} M\right)\right]$ and 1D parameter searching is to estimate radial velocity $\left[O\left(N_{v} M N\right)\right]$. Because the coherent accumulation along the inclined line $F_{\tau_{n}}=$ $-\left(a_{i}+k_{i} t_{n}\right) / \lambda$ can be efficiently implemented by complex addition operations, the total number of complex multiplications of the proposed method is in the order of $\left[O\left(8 M N^{2} \log _{2} N+\right.\right.$ $\left.\left.N^{2} M \log _{2} M+22 M N^{2}+N_{v} M N\right)\right]$.

Suppose $M=400, N_{v}=N_{a}=N_{k}=N$, Fig. 4 shows the computational complexities of the proposed method and other four methods. It can be seen that the proposed method is much more computational efficiently than the GRFT. Although the ACCF iteratively based method has less computational complexity, its detection performance for the maneuvering target with jerk motion is poor as shown in Fig. 3. The ACCF-LVD-based method and the proposed method have approximate computational cost, however, the detection performance of the proposed method is much better than the ACCF-LVD-based method.

According to the above experiment results, we can conclude that among the methods constructing the AFs in the slow time domain, the proposed 2D TAF-based method can achieve a better balance between the computational cost and detection performance. Thus the proposed detection method is suitable for the maneuvering target with jerk motion.

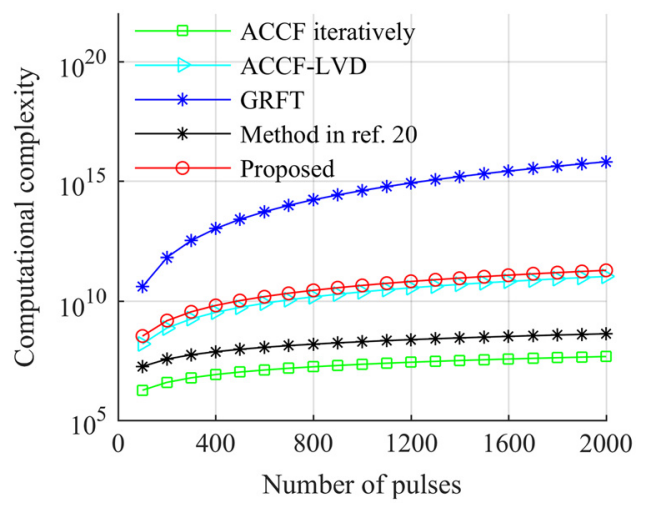

Fig. 4 Computational complexity versus integration pulse number. 


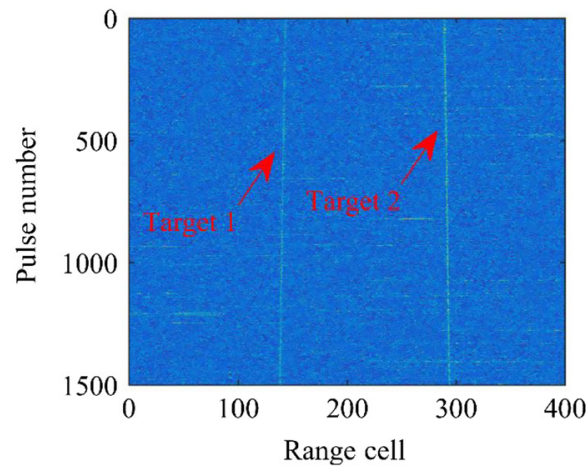

(a)

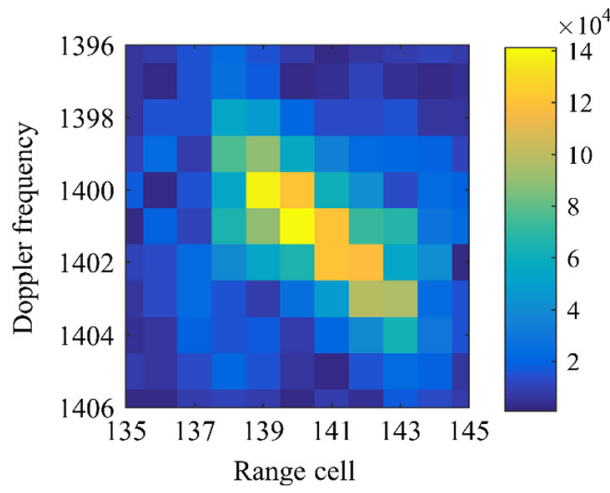

(c)

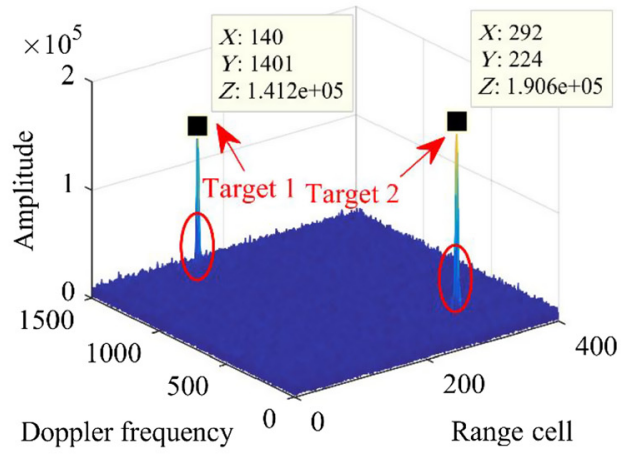

(b)

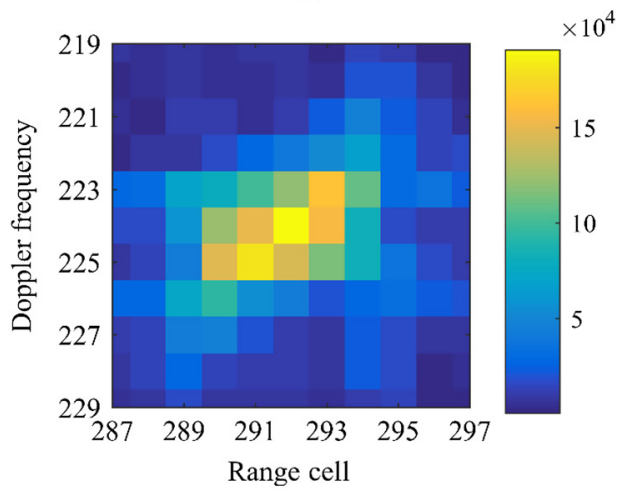

(d)

Fig. 5 Processing results of the real radar data: (a) result after the PC; (b) integration result of the MTD; (c) zoomed into the circled area in (b) for target 1; and (d) zoomed into the circled area in (b) for target 2.

\section{Real Data}

In this section, the raw data are performed to validate the effectiveness of the 2D TAF-based detection method. This radar operates at $L$ wave band for non-cooperation targets, and the other parameters are listed as follows: the bandwidth is $2 \mathrm{MHz}$, the sample frequency is $3.25 \mathrm{MHz}$, PRT is $910 \mu \mathrm{s}$, and the pulse-width is $75 \mu \mathrm{s}$. The integration pulses number is 1500 , and 400 range units are extracted from the collected data.

Figure 5(a) gives the result obtained after PC operation, and it can be seen that the RM appears. After utilizing the MTD, the integration result is shown in Fig. 5(b). To better illustrate the effect of the RM and DFM, Figs. 5(c) and 5(d) magnify the circled areas in Fig. 5(b). It is obvious that the targets' energy spreads in the range-Doppler plane, which will affect the target detection.

After compensating the RM and DFM utilizing the proposed 2D TAF-based method, the coherent integration results are given in Figs. 6(a) and 6(b). For these two targets, The integrated peaks obtained through the proposed method are $2.878 \times 10^{5}$ and $3.028 \times 10^{5}$, respectively, whereas the integrated peaks by the MTD are $1.412 \times 10^{5}$ and $1.906 \times 10^{5}$. Through the zoomed-in images of integrated peaks given in Figs. 6(a) and 6(b), we know the targets' energy has been concentrated.

From the above raw data processing results, we can get the conclusion that the proposed detection method can eliminate the RM and DFM effectively in the realistic application.

\section{Conclusion}

In this paper, we propose a detection method for maneuvering target with jerk motion, the echoes' energy can be integrated into a peak in a 3D parameter space coherently, and the RM and DFM can be compensated simultaneously. Through some numerical experiments and analyses, 


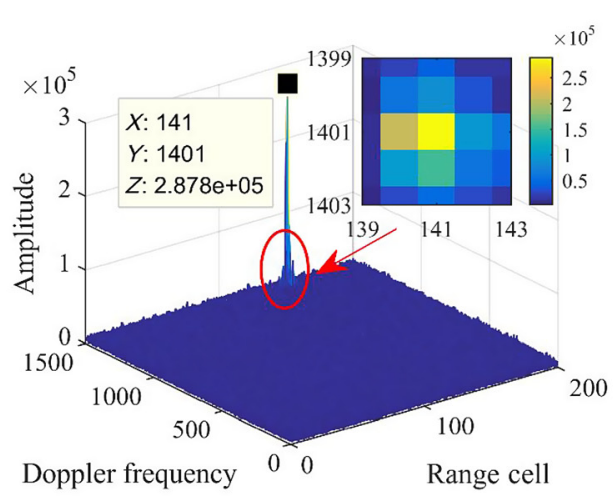

(a)

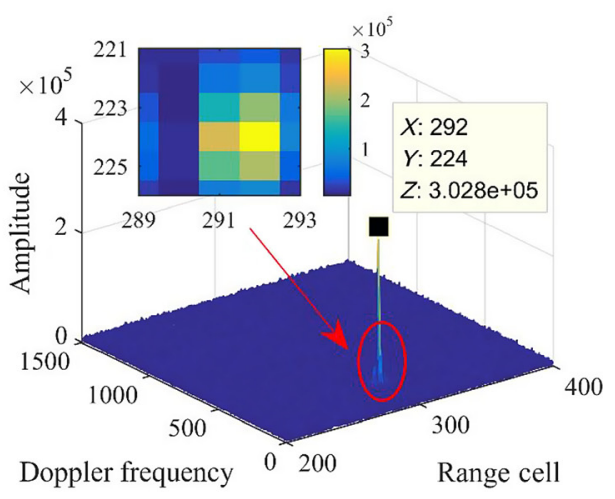

(b)

Fig. 6 Processing results of the real radar data via the proposed method: (a) target 1 and (b) target 2.

we demonstrate that, among the methods defining the AFs in slow time domain, this proposed method can reduce the computational complexity with less detection performance loss. Furthermore, the real measured radar data have been used to verify the effectiveness of the proposed algorithm.

\section{Acknowledgments}

This work was supported in part by the National Natural Science Foundation of China under Grant Nos. 61901372, 62072373, and 61801384; the Natural Science Research Program of Shaanxi Province under Grant No. 2020JQ-599; the Project Funded by China Postdoctoral Science Foundation under Grant No. 2020M683541; and the International Cooperation Foundation of Shaanxi Province under Grant Nos. 2019KW-012 and 2020KW-010.

\section{References}

1. B. D. Carlson, E. D. Evance, and S. L. Wilson, "Search radar detection and track with the Hough transform. Part I: system concept," IEEE Trans. Aerosp. Electron. Syst. 30(1), 102-108 (1994).

2. E. Grossi et al., "A new look at the radar detection problem," IEEE Trans. Signal Process. 64(22), 5835-5847 (2016).

3. X. Huang et al, "Ground-based radar detection for high-speed maneuvering target via fast discrete chirp-Fourier transform," IEEE Access 7, 12097-12113 (2019).

4. J. Zheng et al., "Accurate detection and localization of unmanned aerial vehicle swarmsenabled mobile edge computing system," IEEE Trans. Ind. Inf. 17(7), 5059-5067 (2021).

5. J. Zheng et al., "Efficient data transmission strategy for IIoTs with arbitrary geometrical array," IEEE Trans. Ind. Inf. 17(5), 3460-3468 (2021).

6. J. Zhang et al., "Radar high-speed maneuvering target detection based on joint second-order keystone transform and modified integrated cubic phase function," J. Appl. Remote Sens. 10(3), 035009 (2016).

7. J. Zheng et al., "Parameterized centroid frequency-chirp rate distribution for LFM signal analysis and mechanisms of constant delay introduction," IEEE Trans. Signal Process. 65(24), 6435-6447 (2017).

8. K. Jin et al, "Coherent detection and parameter estimation for radar high-speed maneuvering target based on FAF-LVD," Circuits Syst. Signal Process. 39, 2600-2622 (2020).

9. X. Li et al, "A low complexity coherent integration method for maneuvering target detection," Digital Signal Process. 49, 137-147 (2016).

10. J. Chen et al, "Radar coherent detection for maneuvering target based on product-scaled integrated cubic phase function," Int. J. Antennas Propag. 20198691903 (2019). 
Liang et al.: Fast radar detection method based on two-dimensional trilinear autocorrelation function...

11. X. Chen et al., "Maneuvering target detection via radon-fractional Fourier transform-based long-time coherent integration," IEEE Trans. Signal Process. 62(4), 939-953 (2014).

12. M. I. Skolnik, Radar Handbook, McGraw-Hill, New York (1990).

13. D. Kirkland, "Imaging moving targets using the second-order keystone transform," IET Radar Sonar Navig. 5(8), 902-910 (2011).

14. M. Kevin, C. William, and H. Braham, "The keystone transform: practical limits and extension to second order corrections," in Proc. IEEE Radar Conf., pp. 1264-1269 (2015).

15. J. Xu et al., "Radon-Fourier transform for radar detection. II: blind speed sidelobe suppression," IEEE Trans. Aerosp. Electron. Syst. 47(4), 2473-2489 (2011).

16. J. Yu et al., "Radon-Fourier transform for radar detection. III: optimality and fast implementations," IEEE Trans. Aerosp. Electron. Syst. 48(2), 991-1004 (2012).

17. J. Zheng et al., "Radar high-speed target detection based on the scaled inverse Fourier transform," IEEE J. Sel. Top. Appl. Earth Obs. Remote Sens. 8(3), 1108-1119 (2015).

18. J. Zheng et al., "Radar high-speed target detection based on the frequency-domain derampkeystone transform," IEEE J. Sel. Top. Appl. Earth Obs. Remote Sens. 9(1), 285-294 (2016).

19. J. Xu et al., "Radar maneuvering target motion estimation based on generalized RadonFourier transform," IEEE Trans. Signal Process. 60(12), 6190-6201 (2012).

20. J. Zhang et al, "Novel fast coherent detection algorithm for radar maneuvering target with jerk motion,” IEEE J. Sel. Top. Appl. Earth Obs. Remote Sens. 10(5), 1792-1803 (2017).

21. X. Li et al., "Fast non-searching method for maneuvering target detection and motion parameters estimation," IEEE Trans. Signal Process. 64(9), 2232-2244 (2016).

22. D. Carlson, D. Evance, and L. Wilson, "Search radar detection and track with the Hough transform. Part II: detection statistic," IEEE Trans. Aerosp. Electron. Syst. 30(1), 109-115 (1994).

Zhongying Liang received her BS and MS degrees from Xidian University in 2008 and 2011, respectively. Since 2017, she has worked toward her $\mathrm{PhD}$ in School of Electronics and Information, Northwestern Polytechnical University, Xi'an, China. Her research interests include radar signal processing and target detection.

Yanyan Li received her MS and $\mathrm{PhD}$ degrees in signal and information processing from Xidian University, Xi' an, China, in 2011 and 2016, respectively. Currently, she is an associate professor with the School of Information Science and Technology, Northwest University, Xi'an. Her research interests include ISAR imaging, target detection and time-frequency analysis.

Biographies of the other authors are not available. 